\title{
Workpiece Placement Optimization in Robotic-based Manufacturing
}

\author{
S. Caro* C. Dumas ${ }^{* *}$ S. Garnier ${ }^{* * *}$ B. Furet ${ }^{* * * *}$ \\ * IRCCyN/CNRS, Nantes, France \\ (e-mail: stephane.caro@irccyn.ec-nantes.fr) \\ ** IRCCyN/University of Nantes, Nantes, France \\ (e-mail: claire.dumas@univ-nantes.fr) \\ *** IRCCyN/University of Nantes, Nantes, France \\ (e-mail: sebastien.garnier@univ-nantes.fr) \\ **** IRCCyN/University of Nantes, Nantes, France \\ (e-mail: benoit.furet@univ-nantes.fr)
}

\begin{abstract}
Roboticists are faced with new challenges in robotic-based manufacturing. Up to now manufacturing operations that require both high stiffness and accuracy have been mainly realized with computer numerical control machine tools. This paper aims to show that manufacturing finishing tasks can be performed with robotic cells knowing the process cutting conditions and the robot stiffness throughout its Cartesian workspace. It makes sense that the finishing task of large parts should be cheaper with robots. However, machining robots have not been adapted for such operations yet. As a consequence, this paper introduces a methodology that aims to determine the best placement of the workpiece to be machined knowing the elastostatic model of the robot and the cutting forces exerted on the tool. Therefore, a machining quality criterion is proposed and an optimization problem is formulated and solved. The KUKA KR270-2 robot is used as an illustrative example throughout the paper.
\end{abstract}

Keywords: Robotic manipulators, Machining, Quality, Optimization, Workpiece placement.

\section{INTRODUCTION}

Industrial robots were originally dedicated to "pick and place" operations. They start to be used for machining operations such as trimming, deflashing, degating, sanding and sawing. Machining robots are currently developed to help the operator realize machining operations, which have been mainly performed with Computer Numerical Control (CNC) machines so far. Industrial robots can be used, first to reduce scrap rates and production costs, and secondly to increase the volume and flexibility of production lines. However, industrial robots are not as accurate as CNC machine tools, but the total cost of a machining robot is $30 \%$ less compared with an equivalent CNC machine. Therefore, it makes sense that the larger the parts to be machined, the more interesting robot machining.

This paper introduces a methodology that aims to determine the best placement of the workpiece to be machined knowing the cutting forces exerted on the tool and the elastostatic model of the robot. A machining quality criterion is proposed and an optimization problem is formulated and solved. The KUKA KR270-2 robot is used as an illustrative example throughout the paper.

Section 2 presents the workpiece test and the cutting forces applied by the workpiece on the tool along the machined

\footnotetext{
* This work was partially supported by the Agence Nationale de la Recherche (ANR), France (Project ANR-2010-SEGI-003- 02COROUSSO).
}

path. Section 3 describes the robotic cell. Section 4 deals with the formulation of a mono-objective optimization problem to find the optimum placement of the workpiece with regard to a proposed machining quality criterion. Section 5 highlights the optimum and worst workpiece placements within the robotic cell. The best and worst redundancy planning schemes associated with those placements are also determined. Section 6 is about the conclusions of the paper and the future work.

\section{MACHINING TASK}

Figure 1 illustrates the workpiece to be machined, which is made up of aluminum alloy. $\mathcal{F}_{W}$ of origin $O_{W}$ is the frame attached to the workpiece. The five segments $A B$, $B C, C O_{W}, O_{W} D$ and $D E$, of length equal to $200 \mathrm{~mm}$ each, have to be milled. The tool path is offset by the tool radius from the five segments to be milled. The tool path is discretized into $n$ points and is shown in dashed line in Fig. 1. Frame $\mathcal{F}_{P i}$ is attached to the $i$ th point of the tool path, $i=1, \ldots, n . X_{P i}$ is along the feed direction. $Z_{P i}$ is along the tool axis and points toward the robot.

Table 1. Cutting Conditions

\begin{tabular}{ccccc}
\hline \hline Spindle speed & Feed speed & $f_{z}$ & $a_{p}$ & $a_{e}$ \\
$20000 \mathrm{rpm}$ & $4 \mathrm{~m} / \mathrm{min}$ & $0.05 \mathrm{~mm}$ & $5 \mathrm{~mm}$ & varies \\
\hline \hline
\end{tabular}

The machining quality is affected by the robot deviation due to the cutting forces applied on the tool Dumas et al. (2012). The cutting conditions are given in Tab. 1 where $f_{z}, a_{p}$ and $a_{e}$ denote the feed rate, the depth of cut and the 


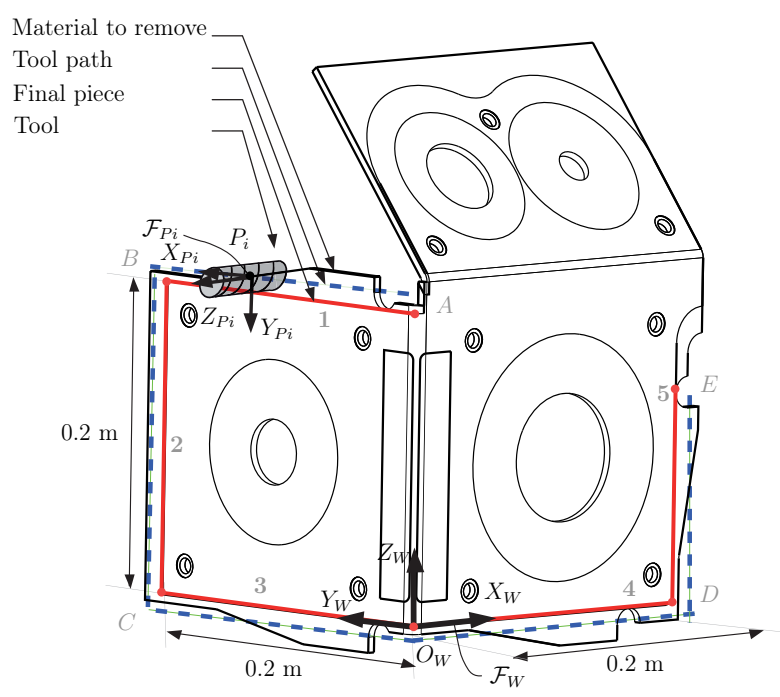

Fig. 1. Workpiece

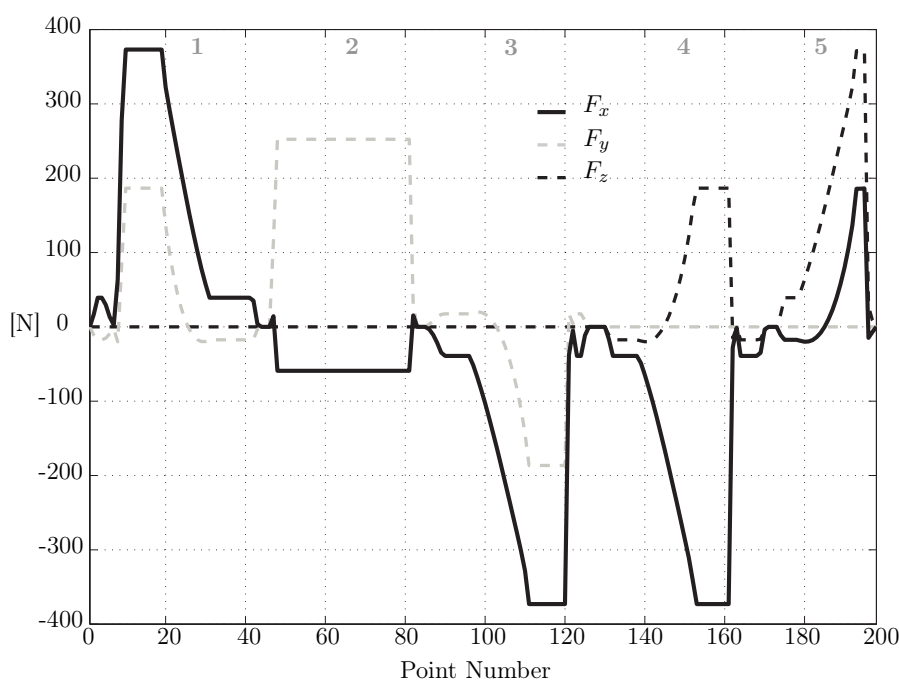

Fig. 2. Force components applied by the tool on the workpiece expressed in frame $\mathcal{F}_{W}$ as a function of the tool path point number

width of cut, respectively. The cutting forces are evaluated thanks to the cutting force model described in Tlusty et al. (1975). As a result, Fig. 2 depicts the force components applied by the tool on the workpiece along axes $X_{W}, Y_{W}$ and $Z_{W}$ of frame $\mathcal{F}_{W}$ as a function of the tool path point number.

\section{ROBOTIC CELL AND PARAMETERIZATION}

The robotic cell shown in Fig. 3 is composed of a KUKA KR270-2 robot, a FISCHER milling spindle mounted on the robot end-effector and a rotary table.

The geometric parameters of the robot are presented in Dumas et al. (2011a). An identification procedure was developed in Dumas et al. (2011b) in order to determine all joint stiffness values of the KUKA KR270-2 robot. Accordingly, the translational and rotational displacements of the tool can be predicted knowing the wrench exerted on it. It is noteworthy that the flexibility of the links is considered through the identified joint stiffness values.

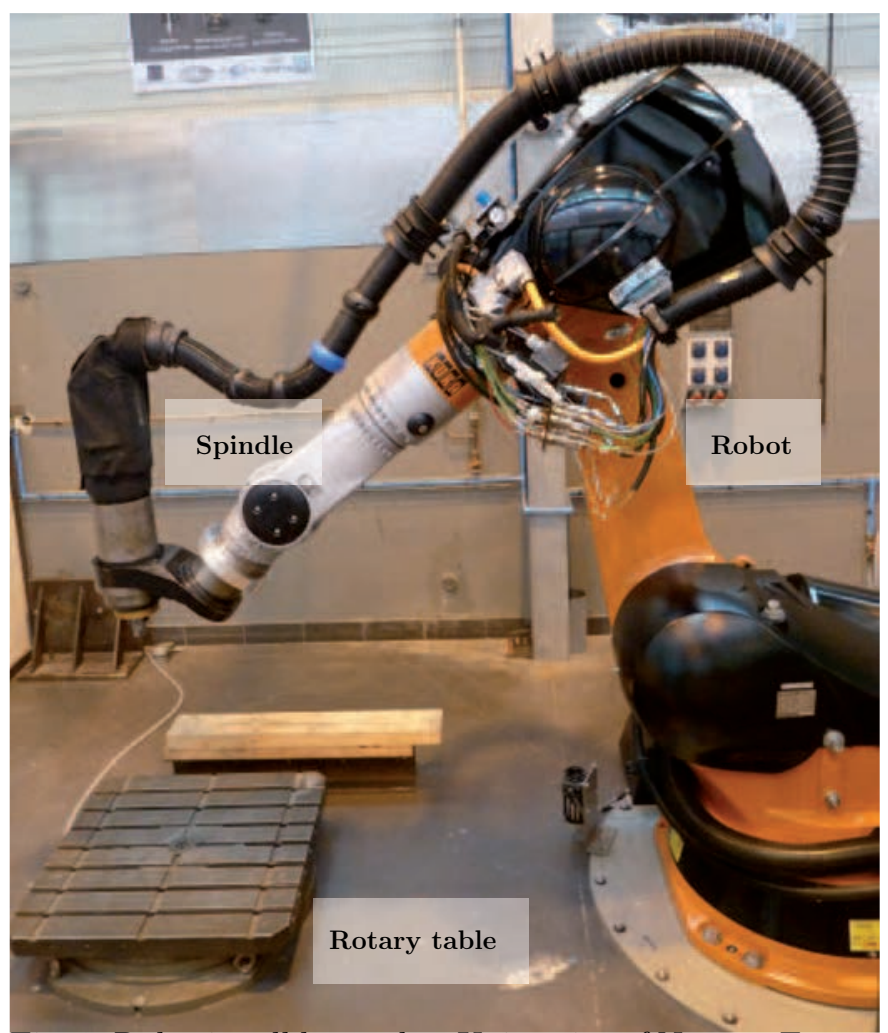

Fig. 3. Robotic cell located at University of Nantes, France

Table 2. Joint Stiffness Values of KUKA KR270-2 Robot in [MNm/rad]

\begin{tabular}{cccccc}
\hline \hline$k_{\theta_{1}}$ & $k_{\theta_{2}}$ & $k_{\theta_{3}}$ & $k_{\theta_{4}}$ & $k_{\theta_{5}}$ & $k_{\theta_{6}}$ \\
0.237 & 3.32 & 2.79 & 0.486 & 0.521 & 0.38 \\
\hline \hline
\end{tabular}

The joint stiffness values of the KUKA KR270-2 robot are given in Tab. 2. $k_{\theta_{l}}$ is the $l$ th joint stiffness value, $l=1, \ldots, 6$.

The FISCHER milling spindle is of type MFW 1709/24. Its maximum rotational speed is equal to $24000 \mathrm{rpm}$ and its power is equal to $20 \mathrm{~kW}$.

The orientation of the workpiece can be fully represented with the variables $\left(Q_{2}, Q_{3}, Q_{4}\right)$, a subset of the quaternions coordinates. Indeed, the quaternions represent the rotations of the workpiece with a rotation axis $\mathbf{u}=$ $\left[\begin{array}{lll}u_{x} & u_{y} & u_{z}\end{array}\right]^{T}$ and an angle $\theta$. The relation between the quaternions and the axis and angle representation can be found in Khalil et al. (2002):

$$
\begin{aligned}
& Q_{1}=\cos (\theta / 2) \\
& Q_{2}=u_{x} \sin (\theta / 2) \\
& Q_{3}=u_{y} \sin (\theta / 2) \\
& Q_{4}=u_{z} \sin (\theta / 2)
\end{aligned}
$$

where $u_{x}^{2}+u_{y}^{2}+u_{z}^{2}=1$ and $0 \leq \theta \leq \pi$.

Nevertheless, the workpiece can be only rotated about the vertical axis of the robot base frame $\mathcal{F}_{0}$ due to the geometry of the rotary table. Therefore,

$$
Q_{2}=Q_{3}=0
$$

and

$$
-1 \leq Q_{4} \leq 1
$$




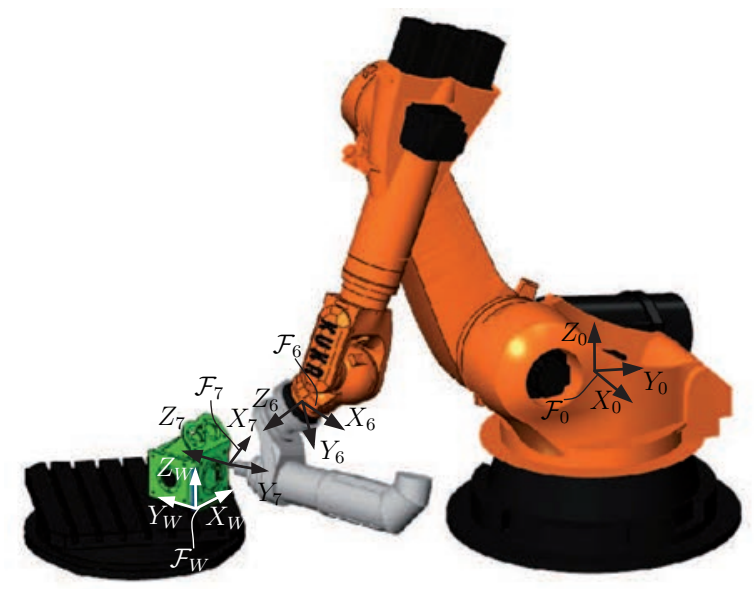

-. Closed loop chain and frames $\mathcal{F}_{0}, \mathcal{F}_{6}, \mathcal{F}_{7}$ and $\mathcal{F}_{W}$

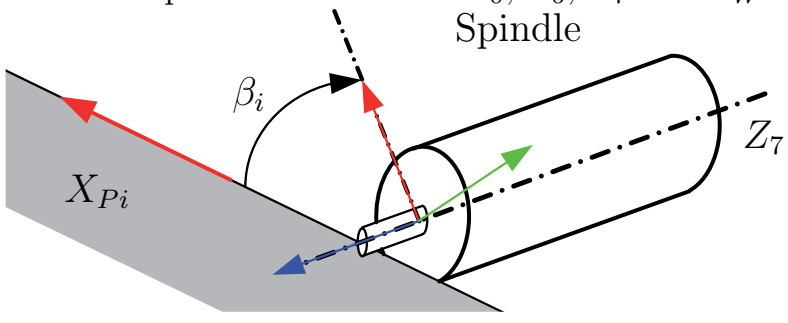

Fig. 5. Kinematic redundancy characterized with angle $\beta_{i}$

Figure 4 represents the closed loop chain composed of the KUKA KR270-2 robot, the FISCHER milling spindle, the workpiece and the rotary table. It also depicts the robot base frame $\mathcal{F}_{0}$, the frame $\mathcal{F}_{6}$ attached to the robot endeffector, the frame $\mathcal{F}_{7}$ attached to the spindle and the workpiece frame $\mathcal{F}_{W}$. From the previous closed loop chain, we can write:

where

$$
{ }^{0} T_{W}{ }^{W} T_{P i}={ }^{0} T_{6}{ }^{6} T_{7}{ }^{7} T_{P i}
$$

${ }^{0} T_{W}$ is the homogeneous transformation matrix from frame $\mathcal{F}_{0}$ to frame $\mathcal{F}_{W}$ expressed as:

$$
{ }^{0} T_{W}=\left[\begin{array}{cccc}
2 Q_{1}^{2}-1 & -2 Q_{1} Q_{4} & 0 & { }^{0} x_{O W} \\
Q_{1} Q_{4} & 2 Q_{1}^{2}-1 & 0 & { }^{0} y_{O W} \\
0 & 0 & 1 & { }^{0} z_{O W} \\
0 & 0 & 0 & 1
\end{array}\right]
$$

${ }^{0} x_{\mathrm{OW}},{ }^{0} y_{\mathrm{OW}}$ and ${ }^{0} z_{\mathrm{OW}}$ being the Cartesian coordinates of point $O_{W}$ expressed in frame $\mathcal{F}_{0}$.

${ }^{W} T_{P i}$ is the homogeneous transformation matrix from frame $\mathcal{F}_{W}$ to frame $\mathcal{F}_{P i}$ attached to the $i$ th point of the tool path as shown in Fig. 1.

${ }^{0} T_{6}$ is the homogeneous transformation matrix from frame $\mathcal{F}_{0}$ to frame $\mathcal{F}_{6}$.

${ }^{6} T_{7}$ is the homogeneous transformation matrix from frame $\mathcal{F}_{6}$ to frame $\mathcal{F}_{7}$ and depends on the geometry of the spindle and how the latter is mounted on the robot endeffector. Here,

$$
{ }^{6} T_{7}=\left[\begin{array}{cccc}
0 & -\sqrt{2} / 2 & \sqrt{2} / 2 & 0 \\
1 & 0 & 0 & 0 \\
0 & \sqrt{2} / 2 & \sqrt{2} / 2 & 0.684 \\
0 & 0 & 0 & 1
\end{array}\right]
$$

${ }^{7} T_{P i}$ is the homogeneous transformation matrix from frame $\mathcal{F}_{P i}$ to frame $\mathcal{F}_{7}$. Note that the KUKA KR270-2 robot has six degrees of freedom, whereas the milling operation sets only five degrees of freedom as the rotation of the spindle about the tool axis is not fixed. Therefore, the robot is redundant with respect to the task and the kinematic redundancy is equal to one as explained in Conkur et al. (1997); Wenger (2004). Here, the kinematic redundancy is characterized by the angle $\beta_{i}$, which corresponds to the rotation angle of the spindle about the tool axis at the $i$ th point of the tool path as shown in Fig. 5. As a consequence,

$$
{ }^{7} T_{P i}=\left[\begin{array}{cccc}
\cos \left(\beta_{i}\right) & \sin \left(\beta_{i}\right) & 0 & 0 \\
\sin \left(\beta_{i}\right) & -\cos \left(\beta_{i}\right) & 0 & 0 \\
0 & 0 & -1 & 0 \\
0 & 0 & 0 & 1
\end{array}\right]
$$

\section{OPTIMUM WORKPIECE PLACEMENT}

This section deals with a methodology that aims to determine the best placement of the workpiece to be machined knowing the cutting forces exerted on the tool and the elastostatic model of the robot. The proposed methodology is highlighted through the definition of a machining quality criterion and the formulation of a mono-objective optimization problem.

\subsection{Machining quality criterion}

It makes sense that the tool displacement $c_{i}$, normal to both the feed direction along axis $X_{P i}$ and the tool axis $Z_{7}$, is mainly responsible for machining errors. ${ }^{0} \mathbf{x}_{P i}$ and ${ }^{0} \mathbf{z}_{7}$ are the unit vectors of axes $X_{P i}$ and $Z_{7}$ expressed in frame $\mathcal{F}_{0}$, respectively. $c_{i}$ can be expressed as follows:

$$
c_{i}=\left|{ }^{0} \delta \mathbf{d}_{i}^{T}\left({ }^{0} \mathbf{z}_{7} \times{ }^{0} \mathbf{x}_{P i}\right)\right|
$$

where ${ }^{0} \delta \mathbf{d}_{i}$ is the point-displacement of the tool at the $i$ th point of the tool path expressed in frame $\mathcal{F}_{0}$ and $\times$ denotes the cross product. The point-displacement of the tool is evaluated at each point of the tool path thanks to the Cartesian stiffness matrix of the KUKA KR270-2 robot obtained in Dumas et al. (2011a) and from the forces applied by the tool on the workpiece that are depicted in Fig. 2.

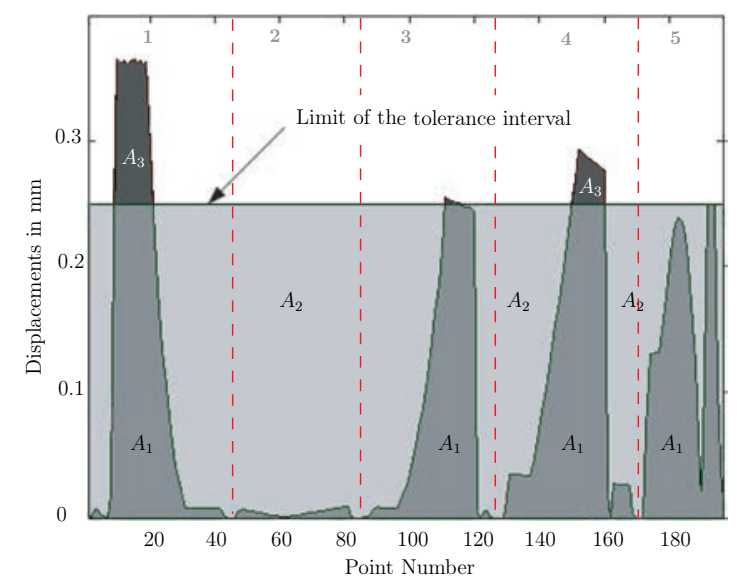

Fig. 6. $c_{i}$ along all five linear paths and definition of the different areas considering the tool displacements and areas $A_{1}, A_{2}$ and $A_{3}$

Figure 6 illustrates the tool displacement $c_{i}$ as a function of the tool path point for a given workpiece placement and redundancy scheme. Figure 6 also depicts the Tolerance 
Interval TI that the geometry of the machined workpiece should respect. Here, the tolerance interval is equal to $0.25 \mathrm{~mm}$. Moreover, the three areas $A_{1}, A_{2}$ and $A_{3}$ are highlighted, namely,

- $A_{1}$ amounts to the sum of the geometric errors $c_{i}$ upper bounded by the prescribed tolerance interval along the tool path;

- $A_{2}$ is the area of the surface, which is lower bounded by the geometric errors $c_{i}$ and upper bounded by the prescribed tolerance interval.

- $A_{3}$ is the area of the surface, which is lower bounded by the tolerance interval and upper bounded by the geometric errors $c_{i}$ that are higher than the tolerance interval.

The machining quality criterion $f_{M Q C}$, which is the objective function of the optimization problem at hand, is defined as:

$$
f_{M Q C}=\frac{\left(w_{1} A_{1}+w_{3} A_{3}\right)}{n} \rightarrow \min
$$

where $w_{1}$ and $w_{3}$ are two weighting factors bounded between 0 and 1 and $n$ is the number of discretized points of the tool path. The values of $w_{1}$ and $w_{3}$ are up to the manufacturer. In the scope of this optimization problem $w_{1}=0.2$ and $w_{3}=0.8$.

The smaller $f_{M Q C}$, the better the machining quality.

\subsection{Decision variables}

The decision variable vector of the optimization problem at hand contains the Cartesian coordinates of point $O_{W}$ expressed in frame $\mathcal{F}_{0}$, i.e., ${ }^{0} x_{O W},{ }^{0} y_{O W}$ and ${ }^{0} z_{O W}$ and the orientation parameter $Q_{4}$ of the workpiece.

The decision variable vector also contains the redundancy planning scheme. Let $\beta_{i}$ be the rotation angle of the spindle about the tool axis at the $i$ th point of the discretized tool path as shown in Fig. 5. Accordingly, the redundancy planning scheme is defined by vector $\boldsymbol{\beta}$ expressed as:

$$
\boldsymbol{\beta}=\left[\begin{array}{llll}
\beta_{1} & \beta_{2} & \cdots & \beta_{n}
\end{array}\right]^{T}
$$

with $-\pi \leq \beta_{i} \leq \pi, i=1, \ldots, n$.

The KUKA KR270-2 robot may have eight solutions to the inverse geometric model, namely, eight postures. The robot has to keep the same posture along a given segment in order to avoid signs of wear. However, the robot posture can change from one segment to the next one. $\mu_{j}$ denotes the solution number to the inverse geometric model of the robot along the $j$ th segment of the tool path, $j=1, \ldots, 5$. As a consequence, the posture scheme is defined by vector $\boldsymbol{\mu}$ expressed as:

$$
\boldsymbol{\mu}=\left[\begin{array}{lllll}
\mu_{1} & \mu_{2} & \mu_{3} & \mu_{4} & \mu_{5}
\end{array}\right]^{T}
$$

with $\mu_{j} \in[1, . ., 8]$.

\subsection{Constraints}

Four types of constraints arise in the optimization problem at hand:

(1) The joint angles of the KUKA KR270-2 robot should be bounded between its joint limits,

$$
\begin{aligned}
-185 \mathrm{deg} & \leq \theta_{1 i} \leq 185 \mathrm{deg} \\
0 & \leq \theta_{2 i} \leq 146 \mathrm{deg} \\
-245 \mathrm{deg} & \leq \theta_{3 i} \leq 29 \mathrm{deg} \\
-350 \mathrm{deg} & \leq \theta_{4 i} \leq 350 \mathrm{deg} \\
-125 \mathrm{deg} & \leq \theta_{5 i} \leq 125 \mathrm{deg} \\
-350 \mathrm{deg} & \leq \theta_{6 i} \leq 350 \mathrm{deg}
\end{aligned}
$$

$\theta_{j i}$ being the $j$ th joint angle of the robot, $j=$ $1, \ldots, 6$, when the tool is located at the $i$ th point of the tool path, $i=1, \ldots, n$. Note that the robot was parameterized by using the modified DenavitHartenberg convention Khalil et al. (2002).

(2) The tool displacement $c_{i}$ expressed with (11) should be smaller than a given value $c_{\max }$, which is defined with regard to the expected machining quality, i.e.,

$$
\max \left(\left|c_{1}\right|,\left|c_{2}\right|, \cdots,\left|c_{n}\right|\right) \leq c_{\max }
$$

where $|$.$| denotes the absolute value.$

(3) The workpiece should be located within a work volume that depends on the rotary table size and location within the robotic cell. Here,

$$
\begin{aligned}
& x_{\text {min }} \leq{ }^{0} x_{O W} \leq x_{\max } \\
& y_{\min } \leq{ }^{0} y_{O W} \leq y_{\max } \\
& z_{\min } \leq{ }^{0} z_{O W} \leq z_{\max }
\end{aligned}
$$

with $x_{\min }=-0.3 \mathrm{~m}, x_{\max }=0.3 \mathrm{~m}, y_{\min }=-2.24 \mathrm{~m}$, $y_{\max }=-1.64 \mathrm{~m}, z_{\min }=-0.602 \mathrm{~m}$ and $z_{\max }=$ $-0.214 \mathrm{~m}$.

(4) The robot should be far from singularities while the tool follows the path, namely,

$$
1 / \kappa_{F}\left(\mathbf{J}_{N i}\right)>0.2, \quad i=1, \ldots, n
$$

where $\mathbf{J}_{N i}$ is the normalized kinematic Jacobian matrix of the KUKA KR270-2 robot defined in Dumas et al. (2011a) and evaluated at the $i$ th point of the tool path. $\kappa_{F}\left(\mathbf{J}_{N i}\right)$ is condition number of matrix $\mathbf{J}_{N i}$ based on the Frobenius norm.

\subsection{Formulation of the optimization problem}

From (12) to (25), the optimization problem to solve in order to find the best workpiece placement can be formulated as follows: 
minimize $f_{M Q C}$

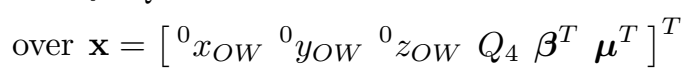

subject to $\max \left(\left|c_{1}\right|,\left|c_{2}\right|, \cdots,\left|c_{n}\right|\right) \leq c_{\max }$

$$
\begin{aligned}
& -185 \mathrm{deg} \leq \theta_{1 i} \leq 185 \mathrm{deg} \\
& 0 \leq \theta_{2 i} \leq 146 \mathrm{deg} \\
& -245 \mathrm{deg} \leq \theta_{3 i} \leq 29 \mathrm{deg} \\
& -350 \mathrm{deg} \leq \theta_{4 i} \leq 350 \mathrm{deg} \\
& -125 \mathrm{deg} \leq \theta_{5 i} \leq 125 \mathrm{deg} \\
& -350 \mathrm{deg} \leq \theta_{6 i} \leq 350 \mathrm{deg} \\
& 1 / \kappa_{F}\left(\mathbf{J}_{N i}\right)>0.2 \\
& x_{\text {min }} \leq^{0} x_{O W} \leq x_{\text {max }} \\
& y_{\text {min }} \leq^{0} y_{O W} \leq y_{\text {max }} \\
& z_{\text {min }} \leq^{0} z_{O W} \leq z_{\text {max }} \\
& -1 \leq Q_{4} \leq 1 \\
& i=1, \ldots, n
\end{aligned}
$$

Optimization problem (26) aims to find the optimum workpiece placement and the corresponding optimum redundancy planning scheme that minimize the machining quality criterion $f_{M Q C}$ defined by (12) while respecting the set of constraints.

\section{RESULT ANALYSIS}

A hybrid optimization algorithm was used to solve optimization problem (26). As a matter of fact, a genetic algorithm Goldberg (1989) was used to find the initial guess of an interior-point algorithm for large-scale nonlinear programming. The Matlab ga function combined with the Matlab fmincon function wereused.

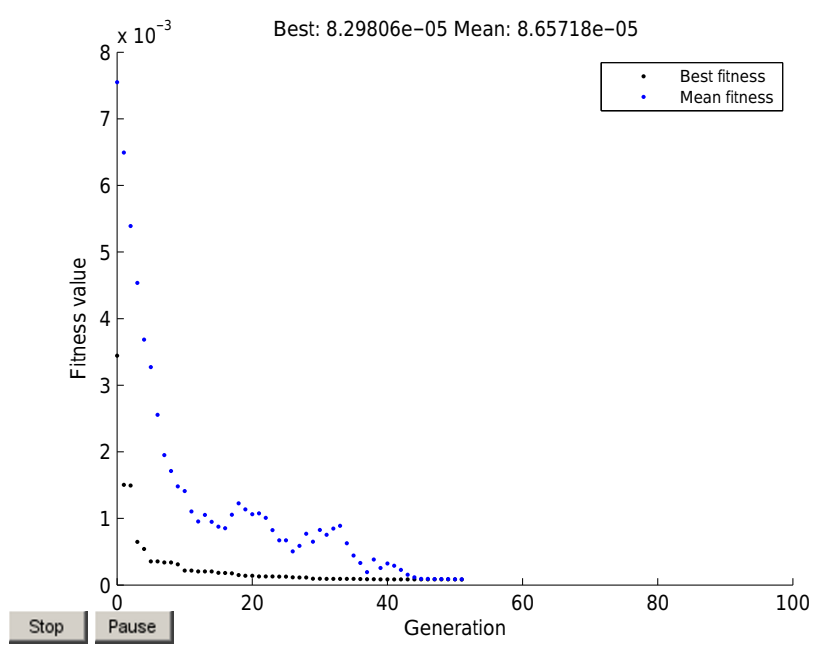

Fig. 7. Convergence of the genetic algorithm for the determination of the best workpiece placement

The genetic algorithm converged after 51 generations as shown in Fig. 7, each population containing 120 individuals. Then, a local optimum decision variable vector $\mathbf{x}_{\text {opt }}$ of optimization problem (26) was obtained with the Matlab fmincon function and is expressed as:

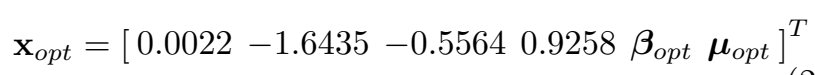

with $\boldsymbol{\mu}_{\text {opt }}=\left[\begin{array}{lllll}4 & 4 & 4 & 5 & 5\end{array}\right]^{T}$ meaning that the robot posture changes between segment 3 and segment 4 only.

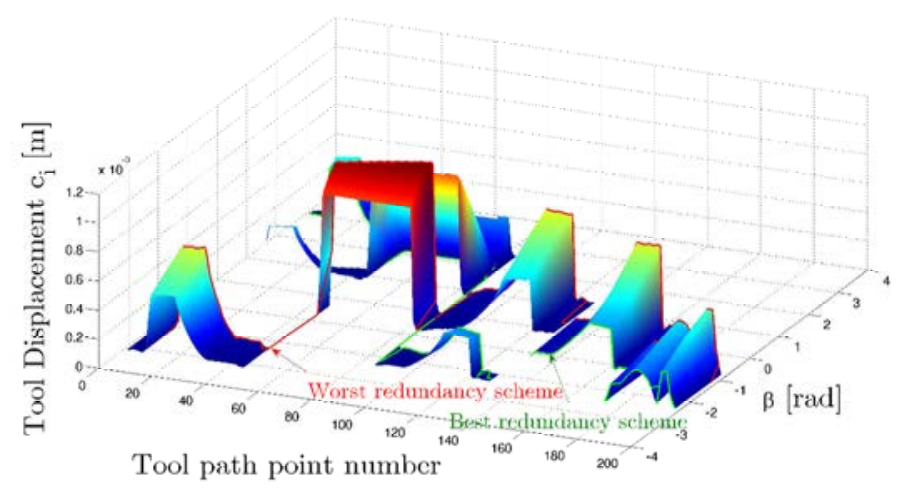

Fig. 8. Tool displacement $c_{i}[\mathrm{~m}]$ as a function of the tool path point number and $\beta$ angle for the best workpiece placement. The green curve characterizes the optimum redundancy planning scheme, i.e., $\boldsymbol{\beta}_{o p t}$ vector, whereas the red curve represents the worst redundancy planning scheme for this workpiece placement

Figure 8 illustrates the tool displacement $c_{i}$ with respect to the tool path point number and $\beta$ angle for the optimal workpiece placement. The green curve characterizes the optimum redundancy planning scheme, i.e., $\boldsymbol{\beta}_{o p t}$ vector, whereas the red curve represents the worst redundancy planning scheme for this workpiece placement. The white areas can not be reached by the robot because of its joint limits.

It is noteworthy that ${ }^{0} x_{O W},{ }^{0} y_{O W},{ }^{0} z_{O W}, Q_{4}$ and $\boldsymbol{\mu}$ are the only decision variables considered by the $g a$ and fmincon functions in this optimization problem solving. As a matter of fact, an optimal $\boldsymbol{\beta}$ vector is searched at each iteration of the genetic algorithm and at each iteration of the interior-point algorithm. This vector is obtained in such a way that it minimizes the objective function $f_{M Q C}$ and avoids discontinuities in the robot joint space along each segment.

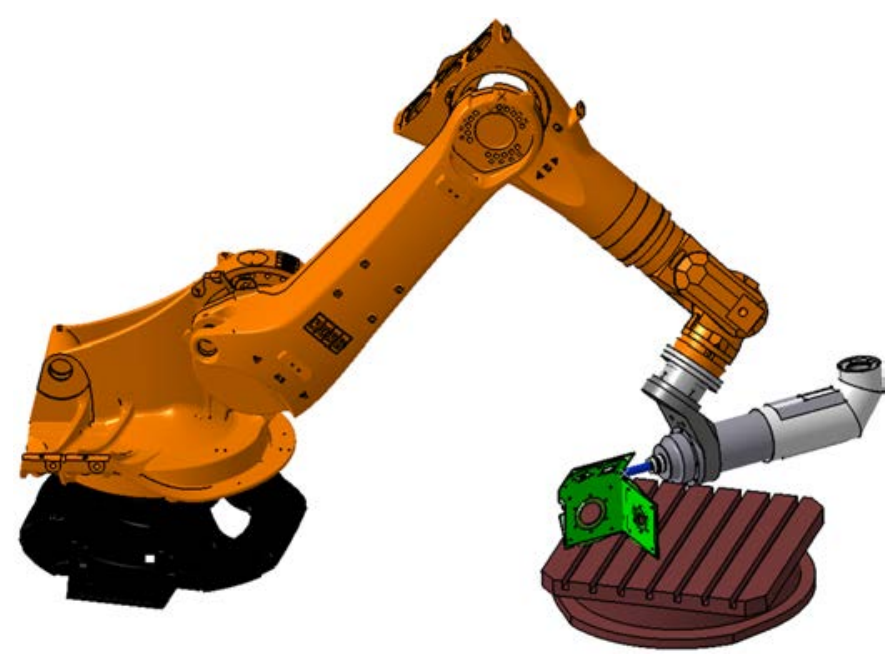

Fig. 9. Optimum workpiece placement 
Figure 9 represents the optimum workpiece placement found by solving optimization problem (26).

It is noteworthy that the worst workpiece placement is obtained by maximizing the objective function $f_{M Q C}$ while respecting the constraints of optimization problem (26).

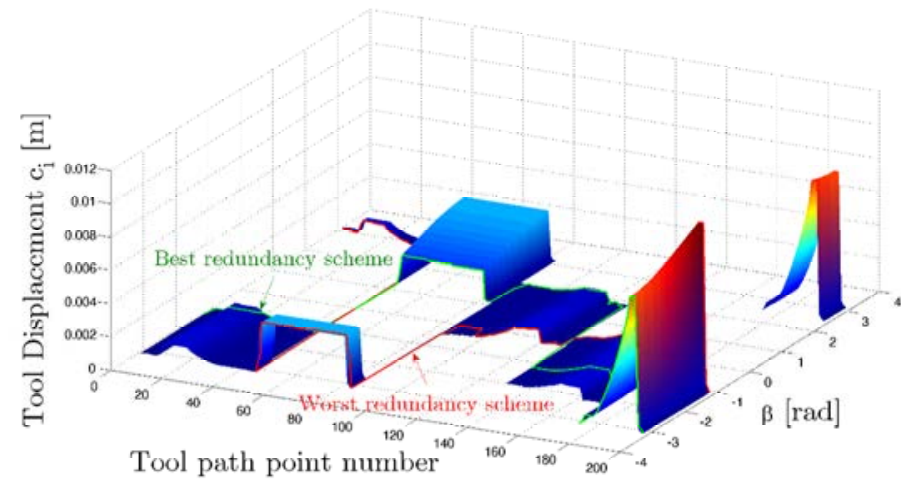

Fig. 10. Tool displacement $c_{i}[\mathrm{~m}]$ as a function of the tool path point number and $\beta$ angle for the worst workpiece placement. The green curve characterizes the optimum redundancy planning scheme, whereas the red curve represents the worst redundancy planning scheme for this workpiece placement

Figure 10 illustrates the tool displacement $c_{i}$ with respect to the tool path point number and $\beta$ angle for the worst workpiece placement. The green curve characterizes the optimum redundancy planning scheme, i.e., $\boldsymbol{\beta}_{\text {opt }}$ vector, whereas the red curve represents the worst redundancy planning scheme for this workpiece placement.

Let us compare the machining quality of the workpiece obtained with the four following milling conditions:

Case 1: Optimum workpiece placement with the best redundancy planning scheme (see Fig. 8)

Case 2: Optimum workpiece placement with the worst redundancy planning scheme (see Fig. 8)

Case 3: Worst workpiece placement with the best redundancy planning scheme (see Fig. 10)

Case 4: Worst workpiece placement with the worst redundancy planning scheme (see Fig. 10)

An video of the milling operation corresponding to Case 1 can be downloaded $a^{1}$. An video of the milling operation corresponding to Case 2 can be downloaded at ${ }^{2}$. An video of the milling operation corresponding to Case 3 can be downloaded $\mathrm{at}^{3}$. An video of the milling operation corresponding to Case 4 can be downloaded at ${ }^{4}{ }^{5}$.

Table 3. Machining Quality Criterion for Four Milling Conditions

\begin{tabular}{ccccc}
\hline \hline & Case 1 & Case 2 & Case 3 & Case 4 \\
$f_{M Q C}[\mathrm{~mm}]$ & 0.083 & 0.35 & 0.82 & 1.2 \\
\hline \hline
\end{tabular}

\footnotetext{
1 http://www.irccyn.ec-nantes.fr/ caro/IFACMIM2013/video1.avi 2 http://www.irccyn.ec-nantes.fr/ caro/IFACMIM2013/video2.avi 3 http://www.irccyn.ec-nantes.fr/ caro/IFACMIM2013/video3.avi 4 http://www.irccyn.ec-nantes.fr/ caro/IFACMIM2013/video4.avi 5 Please, be sure that the tilde " " symbol before "caro" is kept when the previous four links are copied and pasted in your web browser for downloading the videos.
}

Table 3 gives the values of the maching quality criterion $f_{M Q C}$ defined in (12) for the previous four milling conditions. It is apparent that both the workpiece placement and the kinematic redundancy affect the machining quality of the workpiece. Table 3 shows that it is important to pay attention to the workpiece placement and kinematic redundancy. Indeed, $f_{M Q C}$ value for Case 4 is more than 14 times higher than $f_{M Q C}$ value for Case 1.

\section{CONCLUSIONS AND FUTURE WORK}

This paper introduced a methodology to determine the optimum placement of any workpiece to be machined knowing the cutting forces exerted on the tool and the elastostatic model of the KUKA KR270-2 robot. The theoretical results presented in this paper will be checked experimentally soon. The extension of the proposed methodology to robot machining operations with a higher kinematic redundancy of the robot with regard to the task, such as a machining robot mounted on a rail or a mobile platform, are also part of the future work.

\section{ACKNOWLEDGMENT}

The authors would like to acknowledge the financial support of the ANR, France (Project ANR-2010-SEGI-00302-COROUSSO).

\section{REFERENCES}

Ur-Rehman, R., Caro, S., Chablat, D. and Wenger, P. (2010). "Multiobjective Path Placement Optimization of Parallel Kinematics Machines Based on Energy Consumption, Shaking Forces and Maximum Actuators Torques: Application to the Orthoglide," Mechanism and Machine Theory, 45, pp. 1125-1141.

Dumas, C., Caro, S., Garnier, S. and Furet, B. (2012). "Workpiece Placement optimization of Six-revolute Industrial Serial Robots for Machining Operations," The ASME/ESDA 2012 International Conference on Engineering Systems Design and Analysis (ESDA 2012), Nantes, France, July 2-4.

Tlusty, J. and Macneil, P. (1975). "Dynamics of cutting forces in end milling," Annals of the CIRP, 24(1), pp. 21-25.

Dumas, C., Caro, S., Cherif, M., Garnier, S. and Furet, B. (2011). "Joint Stiffness Identification of Industrial Serial Robots," Robotica, Available on CJO 2011 doi:10.1017/S0263574711000932.

Dumas, C., Caro, S., Garnier, S. and Furet, B. (2011). "Joint Stiffness Identification of Six revolute Industrial Serial Robots," Robotics and Computer Integrated Manufacturing, 27(4), pp. 881-888.

Khalil, W. and Dombre, E. (2002). Modeling, Identification and Control of Robots, Hermes Penton Ltd.

Conkur, E.S. and Buckingham, R. (1997). "Clarifying the definition of redundancy as used in robotics," Robotica, 15, pp. 583-586.

Wenger, P. (2004). "Curve-following for redundant manipulators with obstacles : feasibility analysis and solutions," Journal IFToMM Problems of Applied Mechanics, 24(1), No.2, pp. 17-26..

Goldberg, D.E. (1989). Genetic Algorithms in Search, Optimization \& Machine Learning, Addison-Wesley. 